\title{
Expanding the understanding of organic electrochemical transistor function
}

Josefin Nissa, Per Janson, Daniel T Simon and Magnus Berggren

The self-archived postprint version of this journal article is available at Linköping University Institutional Repository (DiVA):

http://urn.kb.se/resolve?urn=urn:nbn:se:liu:diva-173138

N.B.: When citing this work, cite the original publication.

Nissa, J., Janson, P., Simon, D. T, Berggren, M., (2021), Expanding the understanding of organic electrochemical transistor function, Applied Physics Letters, 118(5), 053301.

https://doi.org/10.1063/5.0039345

Original publication available at:

https://doi.org/10.1063/5.0039345

Copyright: American Institute of Physics

http://www.aip.org/ 


\section{Expanding the understanding of organic electrochemical transistor function}

Josefin Nissaa, Per Jansona, Daniel Simona*, Magnus Berggrena

a Laboratory of Organic Electronics, Department of Science and Technology, Linköping University, 60174 Norrköping, Sweden.

*Corresponding author: daniel.simon@liu.se

\section{Abstract}

Organic electrochemical transistors (OECTs) have gained significant interest in recent years due to their ability to transduce and amplify biochemical signals into easily recorded electrical signals. The magnitude of the amplification offered by an OECT is proportional to its transconductance, $g_{\mathrm{m}}$, making $g_{\mathrm{m}}$ an important figure-of-merit. Much attention has therefore been paid to the materials and device geometries which can maximize an OECT's $g_{\mathrm{m}}$. However, less attention has been given to role of the applied potentials and various operational regimes. In this paper we expand on the seminal Bernards and Malliaras model of OECT function to include negative gate potentials, allowing prediction of $g_{\mathrm{m}}$ and general biosensor performance over a broader application range. The expanded model results in five operational regimes, only two of which were covered by the original model. We find an optimal combination of drain and (negative) gate potentials yielding maximal $g_{\mathrm{m}}$. We also find that reducing the pinch-off potential well below the water-splitting limit can yield larger operational windows at the highest $g_{\mathrm{m}}$. Our expanded model presents a general set of guidelines for OECT operation yielding the highest possible $g_{\mathrm{m}}$, and therefore optimal amplification and associated (bio)sensor performance.

Organic electrochemical transistors (OECTs) possess great potential within bioelectronics due to their ability to transduce the chemical signaling used by biological systems to electrical signals that can be recorded and processed by electronic equipment.1-3 The magnitude of the signal amplification offered by an OECT is directly proportional to the transistor's transconductance, making the transconductance an important figure-of-merit. ${ }^{4}$ Here we investigate how the voltage biasing of an OECT influences its transconductance, allowing identification of optimum operational conditions. 
In an OECT, a current flowing through an organic semiconductor channel between the source and drain electrodes is regulated by a potential applied to a third electrode - the gate. The gate and channel are both in contact with an electrolyte, from which ions can enter into the polymer film. In the case of p-type depletion mode OECTs, most commonly exemplified by transistors with a channel comprising poly(3,4-ethylenedioxythiophene) doped with poly(styrenesulfonate) (PEDOT:PSS), dedoping of the channel occurs as cations from the electrolyte enter the channel, replacing charge-carrying holes. ${ }^{5}$ An impeded ion flow into the channel can be detected as a reduction in gating efficacy, a sensing mechanism that has been employed to monitor integrity of biological barriers, such as lipid bilayers ${ }^{6-8}$ and epithelial cell layers 9 ,10. Electroactive cells can also influence the gating of OECTs, making them good alternatives to traditional electrodes for high signal-to-noise recordings of neural activity. ${ }^{11}$

Detection and quantification of chemical compounds is a common application for biosensors. OECTs have been used in fixed-bias settings where the presence of the analyte in the electrolyte alters the drain current as if the gate potential had been changed.12,13 An explanation of the behavior is found in a shift in open circuit potential between the channel and gate, effectively shifting the position of the transistor's transfer curve. ${ }^{14}$ The transfer curve shows how the drain current, at a set drain potential, varies with gate potential. From the transfer curve, the transconductance, $g_{\mathrm{m}}$, can be determined from its slope, $g_{\mathrm{m}}=\partial I_{\mathrm{d}} / \partial V_{\mathrm{g}}$. The transconductance indicates how large the change in drain current is for a given change in gate potential. OECTs have been found to have high transconductances compared to inorganic transistors ${ }^{15}$ and optimization of material parameters and device geometries to yield high transconductance has received a great deal of attention in recent years. ${ }^{16-18}$ In this paper we instead focus on how the biasing of the OECT affects the measured transconductance and propose a method to identify the optimum operational point for any given transistor, in the hopes that our work will help guide the choice of operational parameters to maximize the sensitivity of OECT-based biosensors. We build our analysis upon a framework for steady-state OECT operation introduced by Bernards and Malliaras. ${ }^{19}$

The device model suggested by Bernards and Malliaras (Figure 1) is divided into two interacting circuits, one ionic and one electronic. The ionic circuit describes how a potential applied between channel and gate causes a build-up of ions from the electrolyte in the channel. These injected ions replace the charge carriers in the channel and modulate the charge carrier density, thereby altering the channel conductivity. Figure $1 \mathrm{~b}$ shows the ionic circuit. In the electronic circuit, which describes the current flowing through the channel, the channel conductivity altered by the injected ions together with the applied drain potential determines the drain current. As in the original description of the model we focus on p-type depletion mode transistors, here exemplified 
by a PEDOT:PSS OECT. Later additions to the model have included n-type, as well as accumulation mode OECTs. ${ }^{20}$ Figure 1a shows a schematic of the transistor with source, drain, and gate labeled.

At the core of the electronic circuit model is Ohm's law:

$$
J(x)=q \mu p(x) \frac{d V}{d x}
$$

where $J$ is the current flux, $q$ is the elementary charge, $\mu$ is the hole mobility, $p$ is the hole concentration, and $d V / d x$ is the electric field. In this work we assume constant hole mobility. Dedoping of the channel occurs as ions from the electrolyte enter the channel and replace the charge carriers. The hole concentration in a volume $v$ of the material is then dependent on ions injected from the electrolyte according to:

$$
p=p_{0}\left(1-\frac{Q}{q p_{0} v}\right)
$$

where $p_{0}$ is the initial hole concentration and $Q$ the total charge of injected ions. In steady-state operation the ions injected into a slice $d x$ of the channel is determined by the potential difference between the channel and the electrolyte, and the double layer capacitance per unit area, $c_{\mathrm{d}}$, of the polymer:

$$
Q(x)=c_{d} \cdot W \cdot d x\left(V_{g}-V(x)\right)
$$

In PEDOT the double layer capacitance is thought to extend throughout the bulk of the material and is thus not only dependent on the area under consideration, but also the thickness. Indeed, more recent considerations of PEDOT since the original Bernards and Malliaras model refer to the volumetric capacitance of the material.4,21,22

Combining Eqns. 1-3 gives a governing equation for the current flux through the de-doped regions of the channel:

$$
J(x)=q \mu p_{0}\left(1-\frac{V_{g}-V(x)}{V_{p}}\right) \frac{d V(x)}{d x}
$$

Some important assumptions are made to construct this model. First, the gate either has a significantly larger capacitance than the channel, or is non-polarizable. This is a reasonable assumption as the OECT geometry and/or gate current can easily be arranged to satisfy this criterion. Second, injection of negative ions does not further increase the charge carrier concentration. This is of course an oversimplification as negative gate potentials would introduce 
anions and potentially effect the conductivity in a minor way, but as PEDOT:PSS-based OECTs predominantly operate in depletion mode ${ }^{4}$ this assumption is reasonable to a first approximation. Finally, the model is valid only in the non-faradaic regime, where no electrolysis or other redox processes occur. This final criterion limits the potential range in which the model is accurate.

In the previous work by Bernards and Malliaras ${ }^{19}$, the OECT model was used to find drain currents for both positive and negative drain voltages when gated by a positive gate bias. Here, we exclude the positive drain potentials, but expand the analysis to include negative gate potentials. The expansion into negative gate potentials adds three operational regimes to the two regimes already identified. A chart depicting these five regimes on the $V_{\mathrm{d}}-V_{\mathrm{g}}$ landscape is shown in Figure 2a. Since injection of negative ions is assumed not to influence the carrier concentration, gate potentials more negative than the drain potential do not modulate the conductivity of the channel. In this case, the drain current is determined by the initial conductance of the channel, $G$, and the applied drain potential, $V_{\mathrm{d}}$. We refer to this operational regime as Regime I, and the equation governing the drain current is:

$$
I=G V_{d}
$$

In Regime II, the hole concentration remains unaffected in the parts of the channel with potentials above the gate potential. Between the point where $V(x)=V_{\mathrm{g}}$ and the drain, the hole concentration is modulated according to Eqn. 4. Figure $2 \mathrm{~b}$ shows the hole concentration regions along the channel for the suggested operational regimes. Within Regime II the drain current is described by:

$$
I=G\left(V_{d}+\frac{\left(V_{d}-V_{g}\right)^{2}}{2 V_{p}}\right)
$$

where $V_{\mathrm{p}}$ is the pinch-off potential of the channel, which is the positive drain potential causing drain current saturation for $V_{\mathrm{g}}=0 . V_{\mathrm{p}}$ is defined as $q p_{0} T / c_{\mathrm{d}}$. During operation at drain potentials more negative than $-V_{\mathrm{p}}\left(V_{\mathrm{d}}<-V_{\mathrm{p}}\right)$, it is possible for the channel to enter saturation. This regime (Regime III) is similar to Regime II, but close to the drain contact the channel is completely dedoped (depleted). Once a depletion region is established, applying a larger (i.e., more negative) drain potential will not result in a larger drain current. In Regime III, the drain current is described by:

$$
I=G\left(V_{d}^{s a t}+\frac{V_{p}}{2}\right)
$$

where $V_{\mathrm{d}}^{\text {sat }}=V_{\mathrm{g}}-V_{\mathrm{p}}$. 
Since we do not consider positive drain potentials here, moving from negative to positive gate potentials will ensure that the entire channel is at a lower potential than the gate, which expands the modulated charge carrier concentration region all the way to the source electrode. Two operational Regimes, IV and V, are found for gate potentials larger than $0 \mathrm{~V}$, one where the hole concentration is modulated throughout the entire channel (Regime IV), and one where there is a depletion zone closest to the drain (Regime V), leading to saturation in the drain current. These two regimes were previously described by Bernards and Malliaras. The drain current equation for Regime IV is:

$$
I=G\left(1-\frac{V_{g}-\frac{1}{2} V_{d}}{V_{p}}\right) V_{d}
$$

And in Regime $\mathrm{V}$ the drain current is described by

$$
I=-\frac{G V_{d}^{s a t^{2}}}{2 V_{p}}
$$

We proceeded to compare our model with experimental PEDOT:PSS-based OECTs. Briefly, gold $(50 \mathrm{~nm})$ with a titanium adhesion layer $(2 \mathrm{~nm})$ was thermally evaporated on polyethylene naphtalate substrates and photolithographically patterned to define conductors and contacts. PEDOT:PSS (Clevios PH1000) with 5\% v/v ethylene glycol and $0.5 \%$ v/v (3glycidyloxypropyl)trimethoxysilane (GOPS) was spin-coated onto the substrates, yielding a final thickness of about $60 \mathrm{~nm}$. The PEDOT:PSS was patterned using a $\mathrm{CF}_{4} / \mathrm{O}_{2}$ reactive ion etch. $\mathrm{SU}-8$ 2010 (MicroChem) was used as encapsulation material. Transfer curves and output characteristics (Figure S1) were recorded using a Keithley 4200 source meter, starting with the drain potential closest to $0 \mathrm{~V}$ and using a scan rate of $100 \mathrm{mV} / \mathrm{s}$. Modeling of the derived equations in each regime were performed using custom MATLAB software. Drain currents were calculated for 56 drain potentials and 56 gate potentials. Transfer curves were extracted from the resulting 3D surface. Transconductance was determined from the transfer curves, using the built-in MATLAB function diff, by element-wise division of di $f f\left(I_{d}\right)$ with $\operatorname{di} f f\left(V_{g}\right)$.

Figure 3a shows the modeled drain current for an OECT with $V_{\mathrm{p}}=0.9 \mathrm{~V}$ and $G=3.6 \mathrm{mS}$. Values were chosen to match our physical OECT. Modeled and measured transfer curves are shown together in Figure $3 \mathrm{~b}$. The model predicts the shape of the transfer curves well for small drain potentials, but for drain potentials of $-1 \mathrm{~V}$ and lower the experimental curves deviate from the model. This deviation is most clearly pronounced for gate potentials above $-0.2 \mathrm{~V}$, where the magnitude of the current is larger for these two curves than the model predicts. Examination of the residuals (Figure S2a) confirms this picture. The sum of squared residuals (Figure S2b) is 
three times larger for the $V_{\mathrm{d}}=-1 \mathrm{~V}$ transfer curve compared to the value closest below it, and for the $V_{\mathrm{d}}=-1.1 \mathrm{~V}$ transfer curve the increase is eight-fold. The model is only valid in the potential range where no over-oxidation of PEDOT or other electrochemical reactions occur, such as electrolysis of water or reduction of oxygen. During the recording of the lowest drain potential transfer curves the applied potentials were pushed beyond this range. Over-oxidation at the gate can make the gating less effective, and thus make it difficult to limit the drain current and turn the transistor off. Additionally, the drain current is not a measure selective to the current flowing from source to drain through the channel, but will also have components of current due to electrochemical reactions between gate and drain. The gate current can provide information about ongoing electrochemical processes. Indeed, gate currents recorded for high gate potentials applied during measurements of the lowest drain potential transfer curves - i.e., for the largest gate-to-drain potential difference - exceeded $150 \mathrm{nA}$, while the gate currents measured for less negative (smaller) drain potentials typically did not exceed $50 \mathrm{nA}$. Gate currents tended to increase rapidly as soon as a threshold potential value was exceeded, indicating that the current was no longer solely capacitive (Figure S3).

The transconductance, $g_{\mathrm{m}}$, was calculated from the transfer curves. The transconductance is an important figure-of merit for OECTs, describing the transistor's ability to translate a change in gate potential to a change in drain current. Figure 4a shows the transconductances calculated from the experimentally measured transfer curves from Figure $3 \mathrm{~b}$. The corresponding modeled transconductances are presented in Figure 4b. Both the experimental and modeled transconductances show an increasing trend in the maximum transconductance with decreasing (more negative) drain potentials. For drain potentials lower than $-V_{\mathrm{p}}=-0.9 \mathrm{~V}$ there is no further increase in transconductance for the modeled curves, which indeed appeared to be the case for the experimentally obtained transconductances as well. The model suggests two regimes with constant transconductance: Regime IV where the charge carrier concentration is modulated throughout the entire channel and Regime III, the saturation regime for gate potentials below $0 \mathrm{~V}$. For each drain potential, Regime IV starts at $V_{\mathrm{g}}=0 \mathrm{~V}$ and ends at $V_{\mathrm{g}}=V_{\mathrm{d}}+V_{\mathrm{p}}$, where the transistor enters saturation. Regime IV is wide at small drain potentials but becomes narrower as the drain potential approaches $-V_{\mathrm{p}}$ (see Figure $2 \mathrm{a}$ ). Once past $-V_{\mathrm{p}}$, the region of constant transconductance becomes gradually wider again, as the transistor is in saturation. Regime III begins at $V_{\mathrm{g}}=V_{\mathrm{d}}+V_{\mathrm{p}}$ and ends at $V_{\mathrm{g}}=0$. As can be seen in Figure $4 \mathrm{~d}$, the regions of constant transconductance are centered around $V_{\mathrm{g}}=1 / 2 V_{\mathrm{p}}+1 / 2 V_{\mathrm{d}}$, or $V_{\mathrm{g}}=0.45 \mathrm{~V}+1 / 2 V_{\mathrm{d}}$ in this case. Although the experimental transconductances do not display constant regions, with $V_{\mathrm{g}}-1 / 2 V_{\mathrm{d}}$ on the x-axis, the experimental peaks do generally align at $1 / 2 V_{\mathrm{p}}=0.45 \mathrm{~V}$ (Figure $4 \mathrm{c}$ ). Later additions to the Bernards model have 
allowed the charge carrier mobility to vary with concentration, removing regions of constant transconductance and better capturing the shape of the transconductance peaks. ${ }^{23}$

For the three experimental curves recorded at the largest drain potentials, $-0.9 \mathrm{~V}$ to $-1.1 \mathrm{~V}$, the peak position shifts away from $1 / 2 V_{\mathrm{p}}$. With the $V_{\mathrm{p}}$ of this transistor being $0.9 \mathrm{~V}$, the experimental results' deviation from the model occurred for drain potentials at and below $-V_{\mathrm{p}}$. Since these potentials are sufficiently high to cause electrolysis and are thus outside the range of the model it is difficult to determine whether the model correctly predicts transistor behavior in, mainly, Regime III, but partly also in Regime II. A transistor with a smaller $V_{\mathrm{p}}$ could shed some light on this question since it would be able to operate in these regimes without causing electrochemical reactions. The material and device parameters influencing $V_{\mathrm{p}}$ are initial charge concentration, channel thickness and channel double layer capacitance per unit area. Tailoring these parameters to minimize $V_{\mathrm{p}}$ would be interesting for confirming the model's predictions.

Expanding the Bernards model for OECTs at negative gate potentials allows for prediction of the transconductance peak position and estimation of the transconductance value for transistors with known channel conductance and pinch-off potential. The model suggests that two of the five operational regimes for drain potentials below $0 \mathrm{~V}$ exhibit constant transconductance. In Regime III the transconductance is predicted to be high and constant, which would make operation in this regime especially suitable for amplifying circuits with a wide signal reproduction range. In order to operate in Regime III without causing faradaic reactions, the transistor would need a small $V_{\mathrm{p}}$, since it requires drain potentials to be $-V_{\mathrm{p}}$ or beyond. Experimentally measured transconductances, however, do not display constant regions. Still, the largest transconductance is achieved in Regime III, making it an attractive operational regime if faradaic reactions can be avoided. For transistors with $V_{\mathrm{p}}$ in the potential range where undesired electrochemical reactions occur it is favorable, in terms of transconductance, to use drain potentials as close to $-V_{\mathrm{p}}$ as possible together with gate potentials close to $0 \mathrm{~V}$.

\section{Supplementary Material}

See supplementary material for PEDOT:PSS OECT output characteristics (Figure S1), analysis of modeled vs measure gate currents (Figure S2), and gate current vs gate potential during transfer curve recording (Figure S3). 


\section{Acknowledgements}

This work was primarily funded by the Knut and Alice Wallenberg Foundation. Additional funding was supplied by the Swedish Foundation for Strategic Research, the Swedish Research Council, and the Önnesjö Foundation.

The data that support the findings of this study are available from the corresponding author upon reasonable request.

The authors declare no conflicts of interest.

\section{References}

${ }^{1}$ X. Strakosas, M. Bongo, and R.M. Owens, J. Appl. Polym. Sci. 132, 41735 (2015).

2 D.T. Simon, E.O. Gabrielsson, K. Tybrandt, and M. Berggren, Chem. Rev. 116, 13009 (2016).

3 P. Leleux, J. Rivnay, T. Lonjaret, J.-M. Badier, C. Bénar, T. Hervé, P. Chauvel, and G.G. Malliaras, Adv. Healthc. Mater. 4, 142 (2015).

4 J. Rivnay, S. Inal, A. Salleo, R.M. Owens, M. Berggren, and G.G. Malliaras, Nat. Rev. Mater. 3, 17086 (2018).

5 J.T. Friedlein, R.R. Mcleod, and J. Rivnay, Org. Electron. 63, 398 (2018).

${ }^{6}$ D.A. Bernards, G.G. Malliaras, G.E.S. Toombes, and S.M. Gruner, Appl. Phys. Lett. 89, 53505 (2006).

7 Y. Zhang, S. Inal, C.-Y. Hsia, M. Ferro, M. Ferro, S. Daniel, and R.M. Owens, Adv. Funct. Mater. 26, 7304 (2016).

8 G.C. Faria, D.T. Duong, A. Salleo, C. a. Polyzoidis, S. Logothetidis, J. Rivnay, R. Owens, and G.G. Malliaras, MRS Commun. 4, 189 (2014).

9 J. Rivnay, M. Ramuz, P. Leleux, A. Hama, M. Huerta, and R.M. Owens, Appl. Phys. Lett. 106, (2015). 10 F. Decataldo, M. Barbalinardo, M. Tessarolo, V. Vurro, M. Calienni, D. Gentili, F. Valle, M. Cavallini, and B. Fraboni, Adv. Mater. Technol. 4, 1 (2019).

11 D. Khodagholy, T. Doublet, P. Quilichini, M. Gurfinkel, P. Leleux, A. Ghestem, E. Ismailova, T. Herve, S. Sanaur, C. Bernard, and G.G. Malliaras, Nat. Commun. 4, 1575 (2013).

12 M. Berto, C. Diacci, L. Theuer, M. Di Lauro, D.T. Simon, M. Berggren, F. Biscarini, V. Beni, and C.A. Bortolotti, Flex. Print. Electron. 3, 24001 (2018).

13 F. Cicoira, M. Sessolo, O. Yaghmazadeh, J.A. DeFranco, S.Y. Yang, and G.G. Malliaras, Adv. Mater. 22, 1012 (2009).

${ }^{14}$ D.A. Bernards, D.J. Macaya, M. Nikolou, J.A. DeFranco, S. Takamatsu, and G.G. Malliaras, J. Mater. Chem. 18, 116 (2008). 
15 D. Khodagholy, J. Rivnay, M. Sessolo, M. Gurfinkel, P. Leleux, L.H. Jimison, E. Stavrinidou, T. Hervé, S. bastien Sanaur, R.M. Owens, and G.G. Malliaras, Nat. Commun. 4, 3 (2013).

16 O. Yaghmazadeh, F. Cicoira, D.A. Bernards, S.Y. Yang, Y. Bonnassieux, and G.G. Malliaras, J. Polym. Sci. B Polym. Phys. 49, 34 (2011).

17 M.J. Donahue, A. Williamson, X. Strakosas, J.T. Friedlein, R.R. Mcleod, H. Gleskova, and G.G. Malliaras, Adv. Mater. 30, 1 (2018).

18 H. Sun, M. Vagin, S. Wang, X. Crispin, R. Forchheimer, M. Berggren, and S. Fabiano, Adv. Mater. 30, 1 (2018).

${ }^{19}$ D.A. Bernards and G.G. Malliaras, Adv. Funct. Mater. 17, 3538 (2007).

${ }^{20}$ R. Colucci, H. Frulani, D.P. Barbosa, F. Günther, P. Cavassin, and G.C. Faria, Flex. Print. Electron.

5, (2020).

21 C.M. Proctor, J. Rivnay, and G.G. Malliaras, J. Polym. Sci. Part B Polym. Phys. 54, 1433 (2016).

22 A. V Volkov, K. Wijeratne, E. Mitraka, U. Ail, D. Zhao, K. Tybrandt, J.W. Andreasen, M. Berggren, X. Crispin, and I. V Zozoulenko, Adv. Funct. Mater. 27, 1 (2017).

23 J.T. Friedlein, S.E. Shaheen, G.G. Malliaras, and R.R. Mcleod, Adv. Electron. Mater. 1, 1 (2015). 
Figures
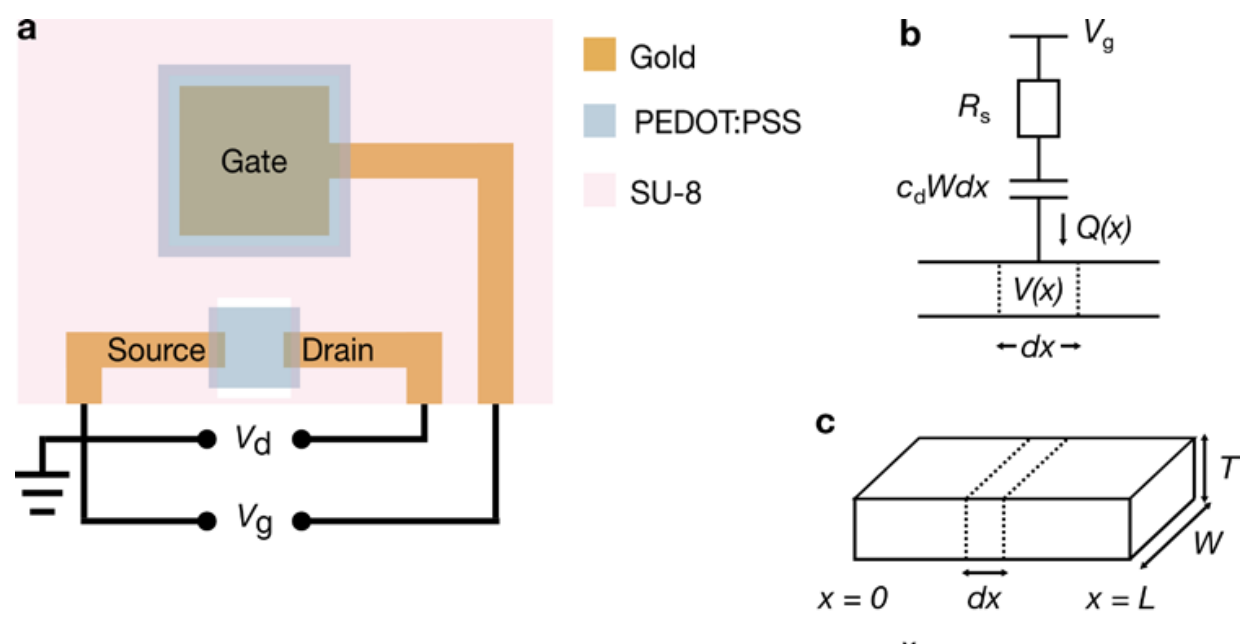

Figure 1. (a) Schematic illustration of a planar OECT and (b) the circuit used to construct the model. $c_{\mathrm{d}}$ represents the capacitance per unit area in the channel. (c) The dimensions of the channel, with $x=0$ at the source and $x=L$ at the drain.

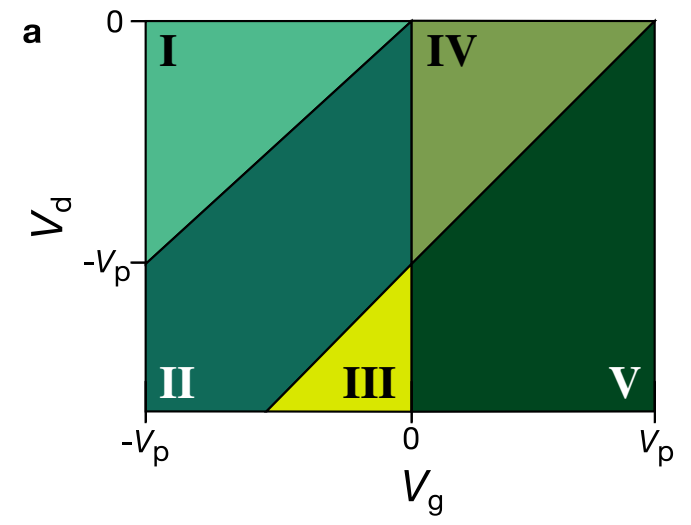

b

$$
\text { I }
$$

I $v(0$

II

III

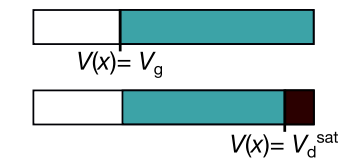

$V_{\mathrm{g}}<0$

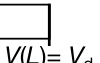

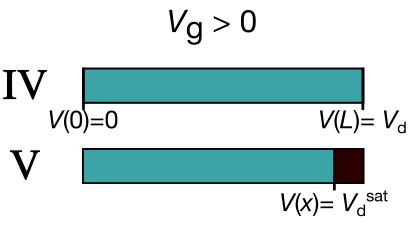

Hole concentration is

$\square$ Intact

$\square$ Modulated

Depleted

Figure 2. a) Color chart displaying the five different OECT operational regimes considered in this work and their locations in the $V_{\mathrm{d}}-V_{\mathrm{g}}$ plane. b) Transistor channel schematic illustrating how three levels of influence on the charge carrier concentration in the channel (intact, modulated, and depleted) combine to constitute the five different operational regimes. 

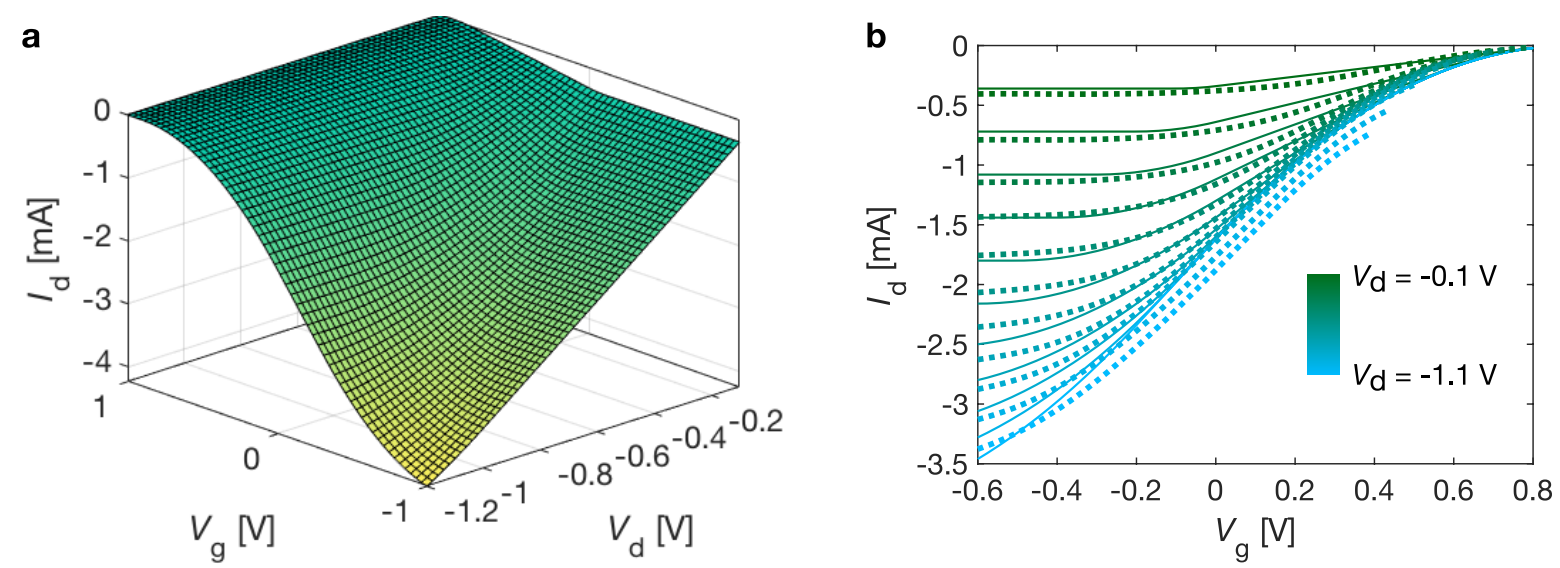

Figure 3. a) Modeled drain current for an OECT with channel conductance, $G=3.6 \mathrm{mS}$, and pinchoff potential, $V_{p}=0.9 \mathrm{~V}$. b) Modeled (solid) and experimentally measured (dotted) transfer curves for drain potentials in the range of -1.1 to -0.1 V. The drain potential step size was $100 \mathrm{mV}$.

a
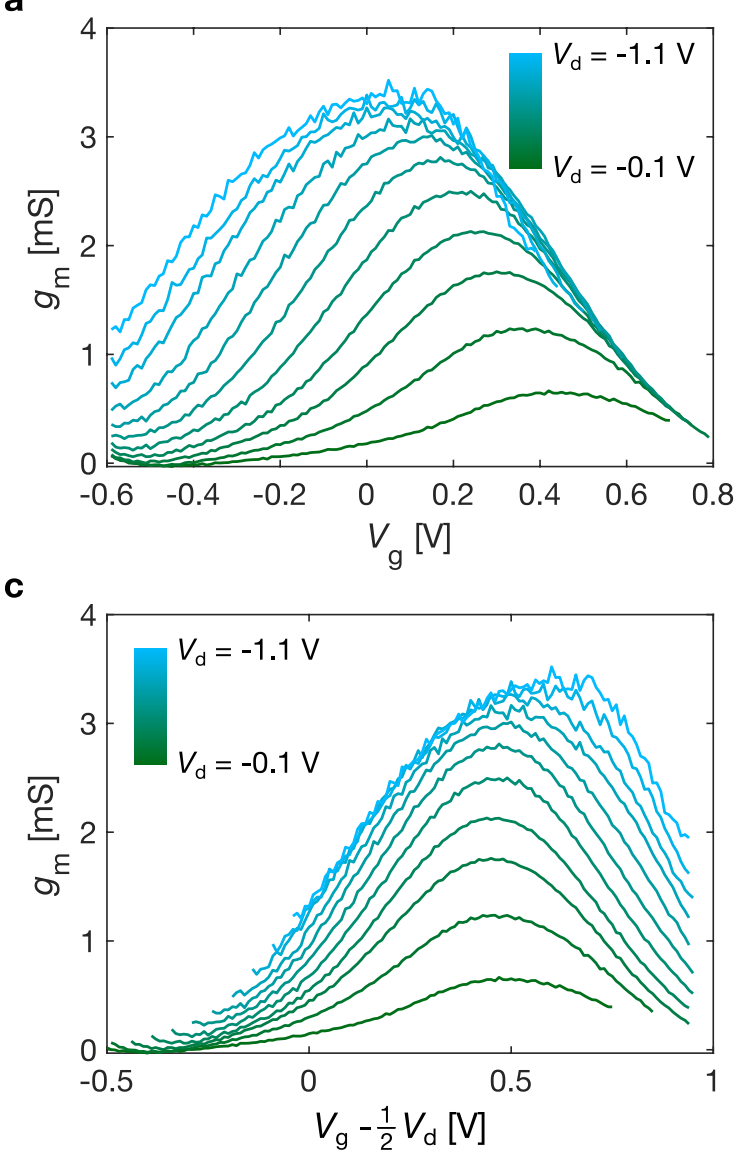

b

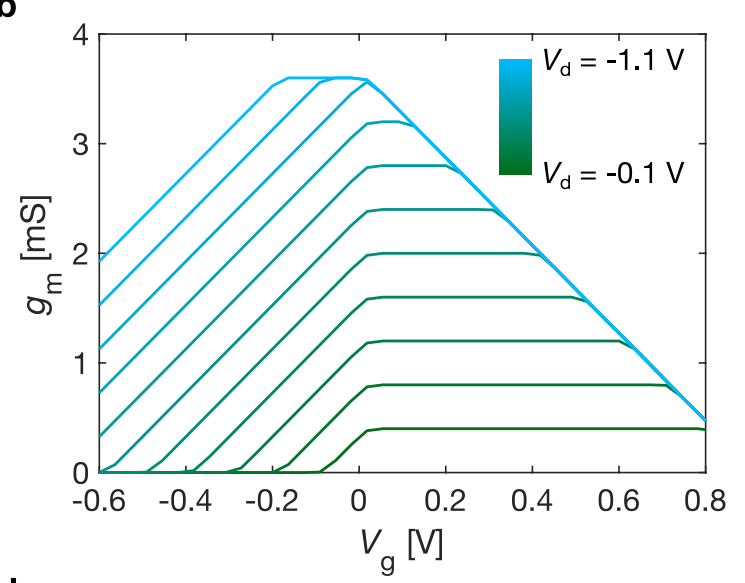

d

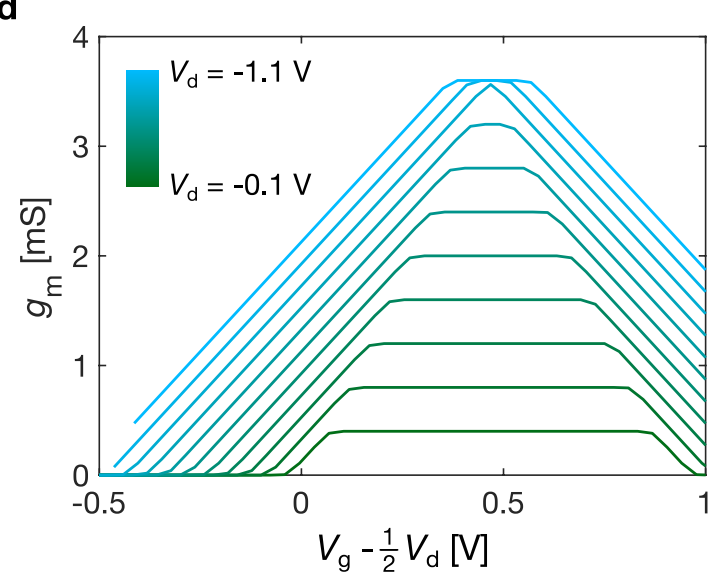

Figure 4. $(a, c)$ Measured and $(b, d)$ modeled transconductances from the transfer curves presented in Figure $3 b$. For $a$ and $b$, the gate potential is on the $x$-axes, while for c and $d$ the $x$-axes are $V_{g}-1 / 2 V_{d}$. The drain potential step size was $100 \mathrm{mV}$. 